ULB-TH-98/02

hep-th/9801053

January 1998

\title{
Statistical Entropy of the Four Dimensional Schwarzschild Black Hole
}

\author{
R. Argurio,日 F. Englert] and L. Houart] \\ Service de Physique Théorique \\ Université Libre de Bruxelles, Campus Plaine, C.P.225 \\ Boulevard du Triomphe, B-1050 Bruxelles, Belgium
}

\begin{abstract}
The entropy of the four dimensional Schwarzschild black hole is derived by mapping it onto a configuration of intersecting branes with four charges. This configuration is obtained by performing several boosts and dualities on a neutral black brane of M-theory to which the Schwarzschild black hole is related by trivial compactification. The infinite boost limit is well-defined and corresponds to extremality where the intersecting brane configuration is a marginal one on which a standard microscopic counting of the entropy can be safely performed. The result reproduces exactly the Bekenstein-Hawking entropy of the four dimensional black hole.
\end{abstract}

\footnotetext{
${ }^{1}$ Aspirant F.N.R.S. (Belgium). E-mail: rargurio@ulb.ac.be

2 E-mail: fenglert@ulb.ac.be

${ }^{3}$ Chercheur I.I.S.N. (Belgium). E-mail: lhouart@ulb.ac.be
} 
Recent progress in string theory and M-theory indicates that an understanding of fundamental issues in black hole physics may well be within reach. Using D-brane techniques a statistical explanation of the entropy for some black holes has been discovered. The entropy has been computed in terms of the degeneracy of D-brane configurations describing in the weak coupling, charged black holes in the extremal and near-extremal limit 迆, 2, 3, 4, 5, 6, 7]. Unfortunately, this systematic approach cannot be applied directly to neutral Schwarzschild black holes. Microscopic considerations based on Matrix theory have however been discussed recently [8, 9, 10, 11, 12, 13]. Other considerations [14] involve a connection between the Schwarzschild black hole and the $2+1$ dimensional BTZ black hole [15, which has been given a microscopic description in 16, 17, 18.

A quantitative analysis of Schwarzschild black holes applying some M-theory concepts has been suggested in [19. It is proposed, following the idea of [20, to view a Schwarzschild black hole as a compactification of a black brane in 11 dimensional supergravity and to relate it to a charged black hole with the same thermodynamic entropy. The charged black hole is obtained by subjecting the black brane to a boost [21] in uncompactified spacetime followed by Kaluza-Klein reduction on a different radius [20] (see also [11]). In [19], a near extremal limit is defined, in which the Schwarzschild radius remains arbitrarily large at infinite boost. It is proposed to use this limit to obtain the entropy of Schwarzschild black holes from the microscopic entropy of the charged ones, viewed as systems of D-branes. This was applied to the seven dimensional black hole, mapped onto a near extremal system of D3-branes.

In this letter we apply this proposal to four dimensional Schwarzschild black holes. In order to relate a four dimensional Schwarzschild black hole to a "countable" D-brane configuration, the procedure is more involved because we will have to perform several boosts and dualities [20]. More precisely, each boost creates a (Ramond-Ramond) charge, and we will end up with a configuration of intersecting branes with four charges. In this case, the infinite boost limit leads exactly to extremality where the configuration is marginal and a standard microscopic counting of the entropy can be safely performed (because it is protected by BPS arguments). In this way the Bekenstein-Hawking entropy of the four dimensional Schwarzschild black hole is exactly recovered.

The metric of a four dimensional Schwarzschild black hole is:

$$
d s^{2}=-f d t^{2}+f^{-1} d r^{2}+r^{2} d \Omega_{2}^{2}, \quad f=1-\frac{r_{0}}{r} .
$$

This metric can be trivially embedded in an 11 dimensional space-time simply by taking its product with a flat 7-dimensional (compact) space. It thus corresponds to a neutral black seven-brane compactified on a $T^{6} \times S^{1}$ characterized by sizes $L_{i}(i=1 \ldots 6)$ and $2 \pi R$. The four and eleven dimensional Newton constants, respectively $G_{4}$ and $G_{11} \equiv l_{p}^{9}$, are related by:

$$
G_{4}=\frac{l_{p}^{9}}{2 \pi L_{1} \ldots L_{6} R} .
$$

The Bekenstein-Hawking entropy of the black hole described by (1) is given by:

$$
S_{B H}=\frac{\pi r_{0}^{2}}{G_{4}}=2 \pi^{2} \frac{L_{1} \ldots L_{6} R}{l_{p}^{9}} r_{0}^{2} .
$$


We now consider this neutral black seven-brane in the framework of M-theory. Most generally, we recall the precise relation between the parameters of M-theory compactified on a circle $S^{1}$ (i.e. the eleven dimensional Planck length $l_{p}$ and the radius $R$ ) and the parameters of type IIA string theory, i.e. the string coupling $g_{s}$ and the string length $l_{s} \equiv \sqrt{\alpha^{\prime}}$ :

$$
\begin{aligned}
R & =g_{s} l_{s}, \\
l_{p}^{3} & =2^{4 / 3} \pi^{7 / 3} g_{s} l_{s}^{3} .
\end{aligned}
$$

This gives the 10 dimensional Newton coupling constant:

$$
G_{10}=\frac{l_{p}^{9}}{2 \pi R}=8 \pi^{6} g_{s}^{2} l_{s}^{8}
$$

Using boosts (in a sense to be defined below) and dualities, we will map the above black brane onto a configuration of intersecting branes carrying 4 Ramond-Ramond charges. We will then show that there exists a limit in which the latter configuration approaches extremality in such a way that the statistical evaluation of its entropy is well-defined.

We now proceed to the careful description of all the steps leading to the final configuration corresponding to the intersection $\mathrm{D} 4 \cap \mathrm{D} 4 \cap \mathrm{D} 4 \cap \mathrm{D} 0$, which is a marginal bound state in the extremal limit.

Let $z$ be a coordinate parametrizing the covering space of the $S^{1}$ factor of the compact space over which the neutral black brane is wrapped. We can now perform a boost of rapidity $\alpha$ in that direction. In the boosted frame, the length $R$ is rescaled to the value:

$$
R^{\prime}=\frac{R}{\cosh \alpha}
$$

We now define a new compactification identifying the boosted coordinate $z^{\prime}$ on intervals of length $2 \pi R^{\prime}$. This is not a constant time compactification as viewed from the unboosted $z$ frame. The 10 dimensional theories defined respectively by compactification on $R$ and $R^{\prime}$ are thus different. They nevertheless coincide at the horizon where all time intervals are blueshifted to zero. The two compactifications are thus on the horizon related by a coordinate transformation, as discussed in [19.

The Schwarzschild black hole embedded in 11 dimensions is of course not invariant under the boost. The metric acquires a non-vanishing off-diagonal $g_{t^{\prime} z^{\prime}}$ component. The resulting configuration, as viewed in 10 dimensions, is a non-extremal set of $\mathrm{D}_{1}$-branes (smeared on the $T^{6}$ ); the subscript indicates that the D0-branes have been created by the first boost. This IIA string theory is characterized by parameters $g_{s}$ and $l_{s}$ which have a well-defined dependence on the boost parameter $\alpha$ given by (4) and (5) when $R$ is replaced by $R^{\prime}$.

In the following step we perform T-dualities in the directions, say, $\hat{1} \hat{2} \hat{3} \hat{4}$ of $T^{6}$ to obtain a non-extremal configuration of $\mathrm{D} 4_{1}$-branes. We use the standard T-duality relations:

$$
L_{i} \rightarrow \frac{4 \pi^{2} l_{s}^{2}}{L_{i}}, \quad g_{s} \rightarrow g_{s} \frac{2 \pi l_{s}}{L_{i}}
$$


We now uplift this IIA configuration to 11 dimensions. Note that this is a "new" M-theory in the sense that the Planck length is now a function of $\alpha$ and the dependence on $\alpha$ of the radius of compactification has changed.

To create a second charge, we perform a boost of parameter $\beta$ on the eleventh direction. Following the same procedure as for the first boost, the radius of compactification of the new M-theory is rescaled by $1 / \cosh \beta$. After compactification, the resulting IIA configuration corresponds to the non-extremal version of the marginal bound state $\mathrm{D} 4_{1} \cap \mathrm{D} 0_{2}$.

We then T-dualize on the $\hat{1} \hat{2} \hat{5} \hat{6}$ directions, leading to a $\mathrm{D} 4_{1} \cap \mathrm{D} 4_{2}$ configuration. The first set of D4-branes lies now in the $\hat{3} \hat{4} \hat{5} \hat{6}$ directions.

Uplifting to eleven dimension for the second time, the parameters now depend on the two boosts $\alpha$ and $\beta$. We are now ready to create a third charge, performing a third boost of parameter $\gamma$.

This results, after compactification and T-dualities over $\hat{1} \hat{2} \hat{3} \hat{4}$, to a non-extremal configuration $\mathrm{D} 4_{1} \cap \mathrm{D} 4_{2} \cap \mathrm{D} 4_{3}$, lying respectively in the $\hat{1} \hat{2} \hat{5} \hat{6}, \hat{3} \hat{4} \hat{5} \hat{6}$ and $\hat{1} \hat{2} \hat{3} \hat{4}$ directions.

A last uplift-boost-compactification procedure characterized by a boost parameter $\delta$ leads to our final configuration $\mathrm{D} 4_{1} \cap \mathrm{D} 4_{2} \cap \mathrm{D} 4_{3} \cap \mathrm{D} 0_{4}$. The corresponding metric in the Einstein frame is (see e.g. [22, 23, 24]):

$$
\begin{aligned}
d s^{2}= & -H_{\alpha}^{-\frac{3}{8}} H_{\beta}^{-\frac{3}{8}} H_{\gamma}^{-\frac{3}{8}} H_{\delta}^{-\frac{7}{8}} f d t^{2}+H_{\alpha}^{-\frac{3}{8}} H_{\beta}^{\frac{5}{8}} H_{\gamma}^{-\frac{3}{8}} H_{\delta}^{\frac{1}{8}}\left(d y_{1}^{2}+d y_{2}^{2}\right) \\
& +H_{\alpha}^{\frac{5}{8}} H_{\beta}^{-\frac{3}{8}} H_{\gamma}^{-\frac{3}{8}} H_{\delta}^{\frac{1}{8}}\left(d y_{3}^{2}+d y_{4}^{2}\right)+H_{\alpha}^{-\frac{3}{8}} H_{\beta}^{-\frac{3}{8}} H_{\gamma}^{\frac{5}{8}} H_{\delta}^{\frac{1}{8}}\left(d y_{5}^{2}+d y_{6}^{2}\right) \\
& +H_{\alpha}^{\frac{5}{8}} H_{\beta}^{\frac{5}{8}} H_{\gamma}^{\frac{5}{8}} H_{\delta}^{\frac{1}{8}}\left(f^{-1} d r^{2}+r^{2} d \Omega_{2}^{2}\right)
\end{aligned}
$$

where

$$
f=1-\frac{r_{0}}{r}, \quad H_{\alpha}=1+\frac{r_{0}}{r} \sinh ^{2} \alpha
$$

and similarly for $H_{\beta}, H_{\gamma}$ and $H_{\delta}$. The non-trivial components of the RR field strengths are:

$$
\begin{aligned}
\tilde{F}_{t y_{1} y_{2} y_{5} y_{6} r} & =-\partial_{r}\left(H_{\alpha}^{-1} \frac{r_{0}}{r} \cosh \alpha \sinh \alpha\right), \\
\tilde{F}_{t y_{3} y_{4} y_{5} y_{6} r} & =-\partial_{r}\left(H_{\beta}^{-1} \frac{r_{0}}{r} \cosh \beta \sinh \beta\right), \\
\tilde{F}_{t y_{1} y_{2} y_{3} y_{4} r} & =-\partial_{r}\left(H_{\gamma}^{-1} \frac{r_{0}}{r} \cosh \gamma \sinh \gamma\right), \\
F_{t r} & =-\partial_{r}\left(H_{\delta}^{-1} \frac{r_{0}}{r} \cosh \delta \sinh \delta\right),
\end{aligned}
$$

where $\tilde{F}_{6}$ is the 10 dimensional Hodge dual of the 4-form RR field strength.

The string coupling and string length of the type $\widehat{\mathrm{IIA}}$ theory in which this configuration is embedded are, in terms of the original quantities appearing in (2) and (3):

$$
\begin{aligned}
& \hat{g}_{s}=4 \pi^{\frac{1}{2}} \frac{l_{p}^{\frac{9}{2}}}{L_{1} L_{2} L_{5} L_{6} R^{\frac{1}{2}}}\left(\frac{\cosh \alpha \cosh \beta \cosh \gamma}{\cosh ^{3} \delta}\right)^{\frac{1}{2}}, \\
& \hat{l}_{s}^{2}=\frac{1}{2^{\frac{4}{3}} \pi^{\frac{7}{3}}} \frac{l_{p}^{3}}{R} \cosh \alpha \cosh \beta \cosh \gamma \cosh \delta \quad\left(=l_{s}^{2} \cosh \alpha \ldots \cosh \delta\right)
\end{aligned}
$$


The lengths of the final 6-torus over which the above configuration is wrapped are:

$$
\begin{aligned}
\hat{L}_{1,2} & =\frac{2^{\frac{2}{3}}}{\pi^{\frac{1}{3}}} \frac{l_{p}^{3}}{R L_{1,2}} \cosh \alpha \cosh \gamma \\
\hat{L}_{3,4} & =L_{3,4} \cosh \beta \cosh \gamma \\
\hat{L}_{5,6} & =\frac{2^{\frac{2}{3}}}{\pi^{\frac{1}{3}}} \frac{l_{p}^{3}}{R L_{5,6}} \cosh \alpha \cosh \beta
\end{aligned}
$$

We now compute the charge densities of the D-branes in this configuration:

$$
\begin{aligned}
Q_{D 4_{1}} & =\frac{1}{16 \pi \hat{G}_{10}} \int_{\hat{T}_{(3,4)}^{2} \times S^{2}} F_{4}=\frac{\pi^{\frac{7}{3}}}{2^{\frac{11}{3}}} \frac{L_{1}^{2} L_{2}^{2} L_{3} L_{4} L_{5}^{2} L_{6}^{2} R^{5} r_{0} \tanh \alpha}{l_{p}^{21} \cosh ^{3} \alpha \cosh ^{3} \beta \cosh ^{3} \gamma \cosh \delta}, \\
Q_{D 4_{2}} & =\frac{1}{16 \pi \hat{G}_{10}} \int_{\hat{T}_{(1,2)}^{2} \times S^{2}} F_{4}=\frac{\pi^{\frac{5}{3}}}{2^{\frac{7}{3}}} \frac{L_{1} L_{2} L_{5}^{2} L_{6}^{2} R^{3} r_{0} \tanh \beta}{l_{p}^{15} \cosh ^{3} \alpha \cosh ^{3} \beta \cosh ^{3} \gamma \cosh \delta}, \\
Q_{D 4_{3}} & =\frac{1}{16 \pi \hat{G}_{10}} \int_{\hat{T}_{(5,6)}^{2} \times S^{2}} F_{4}=\frac{\pi^{\frac{5}{3}}}{2^{\frac{7}{3}}} \frac{L_{1}^{2} L_{2}^{2} L_{5} L_{6} R^{3} r_{0} \tanh \gamma}{l_{p}^{15} \cosh ^{3} \alpha \cosh ^{3} \beta \cosh ^{3} \gamma \cosh \delta}, \\
Q_{D 0_{4}} & =\frac{1}{16 \pi \hat{G}_{10}} \int_{\hat{T}^{6} \times S^{2}} \star F_{2}=\frac{\pi}{2} \frac{L_{1} \ldots L_{6} R r_{0} \sinh \delta}{l_{p}^{9} \cosh \alpha \cosh \beta \cosh \gamma} .
\end{aligned}
$$

The charge densities above are normalized in such a way that the elementary D-branes have a charge density equal to their tension 25.

The tensions of elementary D0 and D4-branes are given by:

$$
\begin{aligned}
& T_{D 0}=\frac{1}{\hat{g}_{s} \hat{l}_{s}}=\frac{\pi^{\frac{2}{3}}}{2^{\frac{4}{3}}} \frac{L_{1} L_{2} L_{5} L_{6} R}{l_{p}^{6}} \frac{\cosh \delta}{\cosh \alpha \cosh \beta \cosh \gamma} \\
& T_{D 4}=\frac{1}{2^{4} \pi^{4} \hat{g}_{s} \hat{l}_{s}^{5}}=\frac{\pi^{\frac{4}{3}}}{2^{\frac{8}{3}}} \frac{L_{1} L_{2} L_{5} L_{6} R^{3}}{l_{p}^{12}} \frac{1}{\cosh ^{3} \alpha \cosh ^{3} \beta \cosh ^{3} \gamma \cosh \delta}
\end{aligned}
$$

Using (15) and (16), we can now compute the different numbers of constituent Dbranes of each type:

$$
\begin{aligned}
& N_{1}=Q_{D 4_{1}} T_{D 4}^{-1}=\frac{\pi}{2} \frac{L_{1} \ldots L_{6} R^{2}}{l_{p}^{9}} r_{0} \tanh \alpha \\
& N_{2}=Q_{D 4_{2}} T_{D 4}^{-1}=(2 \pi)^{\frac{1}{3}} \frac{L_{5} L_{6}}{l_{p}^{3}} r_{0} \tanh \beta \\
& N_{3}=Q_{D 4_{3}} T_{D 4}^{-1}=(2 \pi)^{\frac{1}{3}} \frac{L_{1} L_{2}}{l_{p}^{3}} r_{0} \tanh \gamma \\
& N_{4}=Q_{D 0_{4}} T_{D 0}^{-1}=(2 \pi)^{\frac{1}{3}} \frac{L_{3} L_{4}}{l_{p}^{3}} r_{0} \tanh \delta
\end{aligned}
$$

Strictly speaking, these numbers represent the number of branes only in the extremal limit, but can be interpreted more generally as the difference between the number of 
branes and anti-branes. Note that it is also possible to compute the numbers (17) by evaluating after every boost the number of D0 branes created. Indeed, the numbers are strictly invariant under all the subsequent dualities and further boosts.

We will now show that taking all the boost parameters to infinity with $r_{0}$ kept fixed is equivalent to taking the extremal limit on the intersecting D-brane configuration. In order to do this, we compute the ADM mass:

$$
M=\frac{\pi}{4} \frac{L_{1} \ldots L_{6} R}{l_{p}^{9}} r_{0} \frac{\cosh 2 \alpha+\cosh 2 \beta+\cosh 2 \gamma+\cosh 2 \delta}{\cosh \alpha \cosh \beta \cosh \gamma \cosh \delta} .
$$

This formula is to be compared with the extremal value derived from the charge densities (15):

$$
M_{\text {ext }}=Q_{D 4_{1}} \hat{L}_{1} \hat{L}_{2} \hat{L}_{5} \hat{L}_{6}+Q_{D 4_{2}} \hat{L}_{3} \hat{L}_{4} \hat{L}_{5} \hat{L}_{6}+Q_{D 4_{3}} \hat{L}_{1} \hat{L}_{2} \hat{L}_{3} \hat{L}_{4}+Q_{D 0_{4}} .
$$

It is then easy to show that when all the boost parameters are equal and are taken to infinity, the departure from extremality rapidly goes to zero as:

$$
\frac{M-M_{e x t}}{M_{e x t}} \sim e^{-4 \alpha}
$$

Note that in the same limit both $M$ and $M_{\text {ext }}$ go to zero as $e^{-2 \alpha}$. However we have to take into account the formulas (12) and (13) which tell us that $\hat{g}_{s}$ remains finite and $\hat{m}_{s} \equiv \hat{l}_{s}^{-1}$ goes to zero as $e^{-2 \alpha}$. Thus the masses above are finite in string units. Note also that for all the internal directions the ratio $\hat{L}_{i} / \hat{l}_{s}$ is finite円.

Despite the fact that $\hat{m}_{s}$ is vanishingly small in the limit discussed, we can neglect the massive string modes because the Hawking temperature goes to zero even faster. Indeed, we have:

$$
T_{H}=\frac{1}{4 \pi r_{0} \cosh \alpha \cosh \beta \cosh \gamma \cosh \delta}
$$

which gives a $T_{H} / \hat{m}_{s}$ of order $e^{-2 \alpha}$ in the extremal limit.

For the supergravity description to be valid and not corrected by higher order curvature terms, we have to check that the typical length scale derived from the Riemann tensor is much bigger than the string length. This can indeed be computed, and it holds as soon as $r_{0} \gg l_{p}$.

In this infinite boost limit, the numbers of constituent D-branes (17) tend to a finite value, which moreover is large if we take $r_{0} \gg l_{p}$ as above and we assume that $R$ and all $L_{i}$ are of order $l_{p}$ or bigger. The fact that the $N$ 's are large but tend to a finite value is a crucial element for the validity of our mapping procedure.

We are now in position to compute the microscopic degeneracy of this extremal $\mathrm{D} 4 \cap \mathrm{D} 4 \cap \mathrm{D} 4 \cap \mathrm{D} 0$ configuration. This configuration can indeed be related to the one considered in [4] by a series of T- and S-dualities. One ends up with a configuration consisting of $N_{2}$ D6-branes wrapped on the whole $T^{6}, N_{3}$ NS5-branes lying in the $\hat{1} \hat{2} \hat{3} \hat{4} \hat{5}$ directions, $N_{1}$ D2-branes in the $\hat{5} \hat{6}$ directions (which become actually $N_{1} N_{3}$ after breaking on the NS5-branes) and finally $N_{4}$ quanta of momentum in the $\hat{5}$ direction. Going to flat space,

\footnotetext{
${ }^{1}$ This result and the finiteness of $\hat{g}_{s}$ imply that the string units and the Planck units in 4 dimensions are of the same order in $\alpha$.
} 
the degeneracy of these momentum excitations can be computed as in [4. The statistical entropy is then given for large $N$ 's by:

$$
S_{\text {micro }}=2 \pi \sqrt{N_{1} N_{2} N_{3} N_{4}}
$$

Note that all the $N$ 's, which by (17) are proportional to $r_{0}$, can be taken arbitrarily large because our limit has been defined keeping $r_{0}$ fixed and, in fact, arbitrarily large. Using the values (17) in the infinite boost limit, we find:

$$
S_{\text {micro }}=2 \pi^{2} \frac{L_{1} \ldots L_{6} R}{l_{p}^{9}} r_{0}^{2}=\frac{\pi r_{0}^{2}}{G_{4}},
$$

in perfect agreement with the Bekenstein-Hawking entropy of the original four dimensional Schwarzschild black hole given in (3).

This result calls for some comments.

At first sight, counting states of a Schwarzschild black hole through a mapping onto an extreme BPS black hole seems a very indirect procedure. However the mapping crucially rests on the relation between compactification radii (see e.g. Eq. (7)) ensuring equality of semi-classical thermodynamic entropies. As we have seen, this mapping is equivalent, on the horizon only, to a coordinate transformation in eleven dimensions. The physics outside the horizon is different and in spacetime we have two distinct physical systems. Nevertheless, the very fact that entropy was obtained by a counting of quantum states strongly suggests that the two different systems can be related by a reshuffling of degrees of freedom defined on the horizon.

Another crucial element of our computation is the fast convergence in the infinite boost limit to a configuration of extremal BPS D-branes. This is in sharp contradistinction to the case examined in 19 where all the entropy came from a departure from extremality. This arose because the slow vanishing of the excess mass $\Delta M \equiv M-M_{\text {ext }}$ was exactly compensated by the growth of the internal volume to give a finite value to the entropy of the non-BPS excitations of the D3-branes. In our case the situation is different. The product $\triangle M L$ goes to zero in the limit, leaving a pure BPS state which can then be safely extrapolated to flat space.

\section{References}

[1] A. Strominger and C. Vafa, "Microscopic Origin of the Bekenstein-Hawking Entropy", Phys. Lett. B379 (1996) 99; hep-th/9601029.

[2] C. G. Callan and J. M. Maldacena, "D-brane Approach to Black Hole Quantum Mechanics", Nucl. Phys. B472 (1996) 591; hep-th/9602043.

[3] G. Horowitz and A. Strominger, "Counting States of Near-Extremal Black Holes", Phys. Rev. Lett. 77 (1996) 2368; hep-th/9602051.

[4] J. M. Maldacena and A. Strominger, "Statistical Entropy of Four-Dimensional Extremal Black Holes", Phys. Rev. Lett. 77 (1996) 428; hep-th/9603060. 
[5] C. V. Johnson, R .R. Khuri and R. C. Myers, "Entropy of 4D Extremal Black Holes", Phys. Lett. B378 (1996) 78; hep-th/9603061.

[6] G. T. Horowitz, D. A. Lowe and J. M. Maldacena, "Statistical Entropy of Nonextremal Four-Dimensional Black Holes and U-Duality", Phys. Rev. Lett. 77 (1996) 430; hep-th/9603195.

[7] V. Balasubramanian and F. Larsen, "On D-Branes and Black Holes in Four Dimensions", Phys. Lett. B478 (1996) 199; hep-th/9604189.

[8] T. Banks, W. Fischler, I. R. Klebanov and L. Susskind, "Schwarschild Black Holes from Matrix Theory"; hep-th/9709091.

[9] I. R. Klebanov and L. Susskind, "Schwarzschild Black Holes in Various Dimensions from Matrix Theory"; hep-th/9709108.

[10] M. Li, "Matrix Schwarzschild Black Holes in Large N Limit"; hep-th/9710226.

[11] G. T. Horowitz and E. M. Martinec, "Comments on Black Holes in Matrix Theory"; hep-th/9710217.

[12] T. Banks, W. Fischler, I. R. Klebanov and L. Susskind, "Schwarzschild Black Holes in Matrix Theory II"; hep-th/9711005.

[13] N. Ohta and J.-G. Zhou, "Euclidean Path Integral, D0-Branes and Schwarzschild Black Holes in Matrix Theory"; hep-th/9801023.

[14] K. Sfetsos and K. Skenderis, "Microscopic Derivation of the Bekenstein-Hawking Entropy Fomula for Non-Extremal Black Holes"; hep-th/9711138.

[15] M. Bañados, C. Teitelboim and J. Zanelli, "The Black Hole in Three Dimensional Space-Time", Phys. Rev. Lett. 69 (1992) 1849; hep-th/9204099; M. Bañados, M. Henneaux, C. Teitelboim and J. Zanelli, "Geometry of the $(2+1)$ Black Hole", Phys. Rev. D48 (1993) 1506; gr-qc/9302012.

[16] S. Carlip, "The Statistical Mechanics of the (2+1)-Dimensional Black Hole", Phys. Rev. D51 (1995) 632; gr-qc/9409052; "The Statistical Mechanics of Three Dimensional Euclidean Black Hole", Phys. Rev. D55 (1997) 878; gr-qc/9606043.

[17] A. Strominger, "Black Hole Entropy From Near Horizon Microstates"; hepth/9712251.

[18] D. Birmingham, I. Sachs and S. Sen, "Entropy of Three-Dimensional Black Holes in String Theory"; hep-th/9801019.

[19] F. Englert and E. Rabinovici, "Statistical Entropy of Schwarzschild Black Holes"; hep-th/9801048. 
[20] S. R. Das, S. D. Mathur, S. Kalyana Rama and P. Ramadevi, "Boosts, Schwarzschild Black Holes and Absorption Cross-Sections in M-Theory"; hep-th/9711003.

[21] A. A. Tseytlin, "On the Structure of Composite Black p-brane Configurations and Related Black Holes", Phys. Lett. B395 (1997) 24; hep-th/9611111.

[22] M. Cvetič and A. A. Tseytlin, "Non-Extreme Black Holes from Non-Extreme Intersecting M-branes", Nucl. Phys. B478 (1996) 181; hep-th/9606033.

[23] I. Ya. Aref'eva, M. G. Ivanov and I. V. Volovich, "Non-extremal Intersecting p-branes in Various Dimensions", Phys. Lett. B406 (1997) 44; hep-th/9702079.

[24] N. Ohta, "Intersection Rules for Non-Extreme p-Branes", Phys. Lett. B403 (1997) 218 ; hep-th/9702164.

[25] J. Polchinski, "TASI Lectures on D-Branes"; hep-th/9611050. 